\title{
(ब) \\ Anesthetic challenges in a large multinodular thyroidectomy at a peripheral hospital
}

\author{
Mobeen Ikram ${ }^{1}$, Saira Mahboob ${ }^{2}$
}

${ }^{1}$ Classified Anesthesiologist, ${ }^{2}$ Graded Anesthesiologist Combined Military Hospital, Okara Cantt (Pakistan)

Correspondence: Dr Saira Mahboob, SD-154, Lane No. 6, Askari V, Gulberg III, Lahore (Pakistan); Cell: +92-3344006526; E-mail: saira.mahboob. md@gmail.com

Received: 28 March 2019,

Reviewed: 17, 18 April 2019,

Revised: 4 August,

Reviewed: 22, 26 October 2019,

Accepted: 26 October 2019

\begin{abstract}
We present a case of large multinodular goiter with retrosternal extension scheduled for thyroidectomy under general anesthesia (GA). We anticipated difficult airway due to tracheal compression as well as perioperative hemodynamic instability. She had had hyperthyroidism previously, which had been treated successfully. We planned and prepared to encounter airway problems in the light of Difficult Airway Society guidelines. After counselling and consent, her upper airways were sprayed with local anesthetic solution. Awake, flexible fiberoptic nasal intubation was performed and GA was induced. She had two episodes of bradycardia and hypotension peri-operatively, which were treated with inj atropine. Tracheostomy was performed in anticipation of tracheomalacia, which was de-cannulated on 3rd postoperative day. She was discharged on 4th post-operative day.
\end{abstract}

Key words: Airway Management; Intubation, Intratracheal; Endotracheal; Arrhythmias, Cardiac; Bradycardia; Carotid sinus; Pressoreceptors; Difficult airway; Hypotension; Goiter, Nodular

Citation: Ikram M, Mahboob S. Anesthetic challenges in a large multinodular thyroidectomy at a peripheral hospital. Anaesth pain \& intensive care 2019;23(3):311313

\section{INTRODUCTION}

The incidence of hyperthyroidism has been reported to be $0.8-1.3 \%$ in Europe and the USA. ${ }^{1}$ Peri-operative hemodynamic instability can also be expected in hyperthyroidism, especially if treatment is not optimized pre-operatively. In addition to thyroid storm; peri-operative carotid sinus hypersensitivity may occur peri-operatively. ${ }^{2,3}$ At the same time, difficult intubation is expected in patients with multinodular goiter, especially if it has a retrosternal extension, due to tracheal collapse or deformity. Such cases require adequate planning and preparation to deal with any adverse event. Difficult Airway Society (DAS) has outlined a scheme for dealing with anticipated difficult intubation. Awake fiberoptic intubation is comparatively a safe and effective technique for anticipated difficult airway management. ${ }^{4,5}$ We present the management of such a case of large multinodular goiter with retrosternal extension, which also developed perioperative hemodynamic instability. The patient was successfully managed and discharged home on 4th postoperative day.

\section{CASE REPORT}

A 50 years old, female patient presented to our preanesthesia clinic for assessment and preparation. She had complaints of dyspnea on exertion NYHA class III, orthopnea, dysphagia and a large swelling in front of her neck. She was a known case of multinodular goiter (Figure 1). She had been on oral carbimazole $60 \mathrm{mg}$ and propranolol $20 \mathrm{mg}$ per day for the last one week.

Her baseline investigations (blood complete picture; serum urea, creatinine, ALT, bilirubin) were within normal limits. Her ECG revealed normal sinus rhythm without any evidence of dysrhythmia, ischemia or 


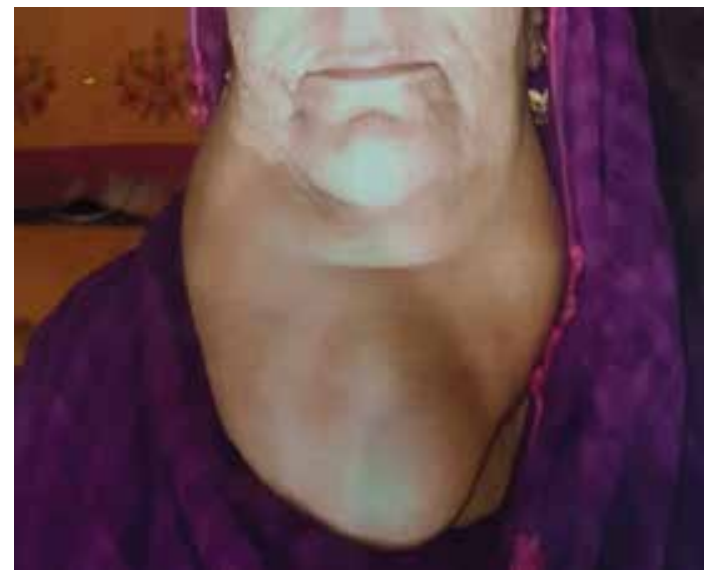

Figure 1: Pre-operative picture showing a large swelling in front of neck (consent of the patient obtained)

infarct. A radiograph chest and neck revealed a large soft tissue mass which had compressing and deviated the trachea (Figure 2).

The surgery was planned once the patient was clinically and chemically euthyroid. Patient was counselled regarding awake flexible fiberoptic endotracheal intubation and the need of invasive blood pressure monitoring. The patient and the attendants were also counselled about the risks of anesthesia and surgery, and the possibility of postoperative tracheostomy and mechanical ventilation. A high risk informed consent was obtained. All medication was continued pre-operatively and blood products were arranged.

On the day of surgery, two wide bore cannulas (14G and18G)were passed. Standard monitoring, including ECG, non-invasive blood pressure, temperature, $\mathrm{SpO}_{2}$ and $\mathrm{EtCO}_{2}$ along with invasive intra-arterial

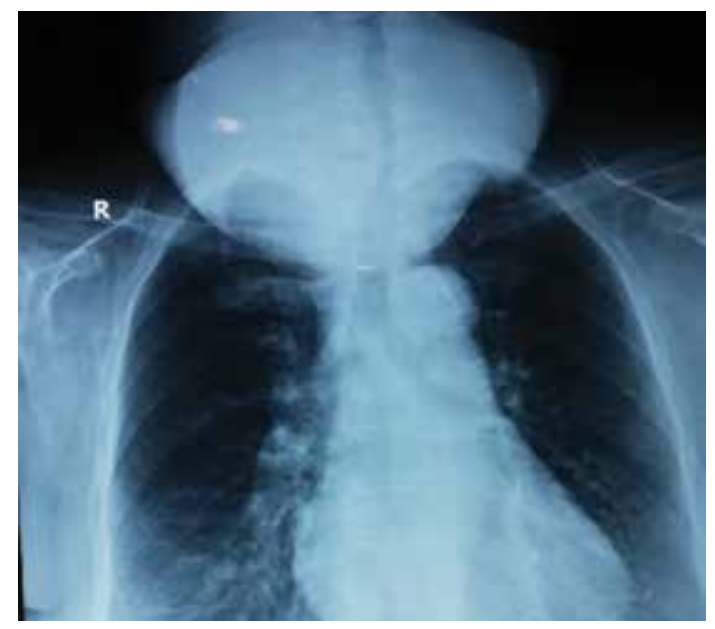

Figure 2: Radiograph of the neck and chest showing tracheal deviation and compression blood pressure (20G cannula in left radial artery), and urine output, was started. After explaining the procedure, patient was given glycopyrrolate $0.2 \mathrm{mg}$ IV, dexamethasone $8 \mathrm{mg}$ and metoclopramide 10 mg. Lignocaine spray $10 \%$ was done for posterior pharyngeal wall. Patient was also told to gargle with 4 $\mathrm{ml}$ of $2 \%$ lignocaine with $10 \mu \mathrm{g}$ adrenaline. Intranasal spray was done with $0.5 \mathrm{ml}$ of $2 \%$ lignocaine. An armored ETT size $6.5 \mathrm{~mm}$ ID was railroaded over Fujinon Fb-120S flexible fiberoptic bronchoscope. Patient was preoxygenated with $100 \%$ oxygen for 3 min and flexible fiberoptic endotracheal intubation was done in the sitting position. Patient required an additional $2 \mathrm{ml}$ of $2 \%$ lignocaine spray at level of vocal cords. The tracheal compression was intubated under vision. Inj propofol $100 \mathrm{mg}$, atracurium 35 $\mathrm{mg}$, and nalbuphine $10 \mathrm{mg}$ were given once correct placement of endotracheal tube was confirmed by bronchoscopic visualization of carina and $\mathrm{EtCO}_{2}$. Surgery proceeded.

She had an episode of severe bradycardia (heart rate $<30 / \mathrm{min}$ ) with hypotension (blood pressure-65/3 $\mathrm{mmHg}$ ) during thyroid manipulation. The surgeon was informed and surgery stopped till it was treated with IV atropine $0.5 \mathrm{mg}$. At the end of surgery, she was extubated once fully awake with return of adequate neuromuscular function. However, she had stridor despite adequate bilateral mobile vocal cords. Tracheostomy was done under general anesthesia for fear of tracheal collapse. Total anesthesia time was nine hours. A total of $5800 \mathrm{ml}$ fluid was administered which included $4000 \mathrm{ml}$ crystalloid and $1200 \mathrm{ml}$ red cell concentrate. Her intra-operative urine output was $800 \mathrm{ml}$ and the estimated blood loss was $1500 \mathrm{ml}$.

She was shifted to surgical intensive care unit when fully awake and had adequate neuromuscular recovery. Patient had a similar episode of sudden onset bradycardia (heart rate $<30 \mathrm{bpm}$ ) and hypotension (blood pressure $50 / 30 \mathrm{mmHg}$ ) at $2 \mathrm{~h}$ postoperatively. Normal saline $500 \mathrm{ml}$ was pushed and atropine 0.5 $\mathrm{ml}$ was administered immediately. She remained hemodynamically stable after that, and was kept on spontaneous mode of mechanical ventilation with pressure support of $10 \mathrm{cmH}_{2} \mathrm{O}, \mathrm{FiO}_{2} 50 \%$ and PEEP $5 \mathrm{cmH}_{2} \mathrm{O}$ overnight. She was weaned off ventilator on 1st postoperative day, tracheostomy tube was removed on 3rd and patient discharged home on 4th postoperatively day.

\section{DISCUSSION}

Our institute is a Class-B hospital with two anesthetists. The patient presented to us in preanesthesia clinic after being refused for surgery and anesthesia at different hospitals due to obvious difficult airway management and issues with perioperative care. She required preoperative 
preparation including optimization of anti-thyroid medication and beta blockade; ${ }^{6,7}$ which were started and continued for three weeks till the patient was clinically and chemically euthyroid.

The associated risks of cardiovascular sequelae, chances of difficult airway, including failed mask ventilation, failed intubation, and the possibility of postoperative tracheal collapse were discussed, and preparations undertaken for dealing any adverse situation. After detailed counselling and a written consent, awake flexible fiberoptic nasal intubation in the sitting position was planned. Local anesthesia of the airway can be achieved by topical spray, nebulization, atomization and nerve blocks. ${ }^{8}$ Nerve blocks include: glossopharyngeal (at tonsillar pillar), superior laryngeal block (at greater cornu of hyoid bone) or translaryngeal block. ${ }^{8,9}$ We used topical lignocaine as well as in spray to anesthetize the airway and orophayrnx. Neither hyoid bone nor trachea were not palpable so superior laryngeal nerve block or trans-tracheal injections were not possible. At the level of epiglottis and vocal cords, we sprayed an additional $2 \mathrm{ml}$ of $2 \%$ lignocaine, and passed the ETT under vision through the tracheal compression.

Our patient had two episodes of sudden onset severe bradycardia and hypotension peri-operatively, which were promptly and timely managed with inj. atropine. Cardiac dysrhythmias are not uncommon in thyroid manipulation during surgery. There have been case reports of similar hemodynamic instability as well as cardiac arrest in thyroid surgery as well as head and neck surgery. ${ }^{10,11}$ This may have been due to carotid sinus hypersensitivity, which has been reported after positioning for thyroidectomy. ${ }^{12}$ Our patient didn't have any pre-operative history of syncope or palpitations nor have any other episode during rest of her hospital stay.

\section{CONCLUSION}

Anesthesia for large multinodular goiter requires a close collaboration between the anesthetist, the surgeon and the endocrinologist. Meticulous preoperative assessment, planning, optimization and preparation and effective communication with the patient are essential tools which must be at hand before embarking upon anesthesia in these patients. Vigilant monitoring, early recognition of complications with prompt management will be required for optimal patient outcomes.

Conflict of interest: None declared by the authors.

Authors' contribution:

MI: Concept, manuscript editing, conduct of the case

SM: Literature review, manuscript writing

\section{REFERENCES}

1. De Leo S, Lee SY and Braverman LE. Hyperthyroidism. Lancet. $2016 ; 388$ (10047):906-18. [PubMed] DOI: 10.1016/S01406736(16)00278-6

2. Akamizu T. Thyroid storm: A Japenense perspective. Thyroid. 2018;28(1):32-40. [PubMed] DOI: 10.1089/thy.2017.0243

3. Mehta $\mathrm{N}$, Abdelmessih $\mathrm{M}$ smith $\mathrm{L}$, Jacoby $D$ and Marieb M. Carotid sinus syndrome as manifestation of head and neck cancer- case report and literature review. Int J Clin Cardiol. 2014;1:012. [Free Full Text]

4. Srivastava D, Dhirraj S. Airway management of a difficult airway due to prolonged enlarged goiter using loco-sedative technique. Saudi J Anaesth. 2013;7(1):86-9. [PubMed] DOI: $10.4103 / 1658-354 X .109829$

5. Shamim F, Yahya M, Ikram M. Awake Fiberoptic intubation in patient with known difficult airway due to huge thyroid goiter. Anaesth Pain Intensive Care. 2017;21(1):94-7. [Eree Full Text]

6. Palace MR. Perioperative management of thyroid dysfunction. Health Serv Insights. 2017;10:1178632916689677. [PubMed] DOI: 10.1177/1178632916689677

7. Ross DS, Burch HB, Cooper DS, Greenlee MC, Laurberg P, Maia $\mathrm{AL}$, et al. 2016 American thyroid association guidelines for diagnosis and management of hyperthyroidism and other causes of thyrotoxicosis. Thyroid. 2016 0ct;26(10):1343421. [PubMed] DOI: 10.1089/ thy.2016.0229

8. Pani N and Rath SK. Regional \& topical anaesthesia of the upper airways. Indian J Anaesth. 2009;53(6):641-8. [PubMed]

9. Reed AP, Han DG. Preparation of the patient for awake fiberoptic

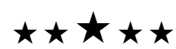

intubation. Anesth Clin North Am. 1991;9:69-81.

10. Nouraei SAR, Davies MR, Obholzer R, Sandhu GS, Porter E. Hemodynamic instability during thyroid surgery: a baroreflex-mediated neurogenic phenomenon? Anaesthesia. 2006;61:282-4. [PubMed] DOl: 10.1111/j.1365-2044.2005.04436.x

11. Chung HS, Park CM, Kim ES, Ghil BG, Park CS. Temporary cardiac arrest in patient under robotically assisted total thyroidectomy causing carotid sinus hypersensitivity- A case report. Korean J Anesthesiol. 2010; 59(Suppl):S137-40. [PubMed] DOI: 10.4097/kjae.2010.59.S.S137

Lilitis E, Papaioannou A, Hatzimichali A, Spyridakis K, Xenaki S, Chalkiadeakis $G$, et al. A case of asystole from carotid sinus hypersensitivity during patient positioning for thyroidectomy. BMC. 2016;16:85. [PubMed] DOI: 10.1186/s12871-016-0255-5 